\title{
DCR2, a Cellular Senescent Molecule, Is a Novel Marker for Assessing Tubulointerstitial Fibrosis in Patients with Immunoglobulin A Nephropathy
}

\author{
Jia Chen ${ }^{a}$ Wei Hu ${ }^{a, b}$ Fei Xiao ${ }^{a}$ Lirong Lin ${ }^{a}$ Kehong Chen ${ }^{a}$ \\ Liming Wang $^{a}$ Xiaoyue Wang ${ }^{a}$ Yani He ${ }^{a}$ \\ ${ }^{a}$ Department of Nephrology, Daping Hospital, Research Institute of Surgery, Army Military \\ Medical University, Chongqing, China; ${ }^{b}$ Department of Nephrology, Army 958 Hospital, \\ Chongqing, China
}

\section{Keywords}

Urinary DcR2 · Cell senescence · Tubulointerstitial fibrosis · Immunoglobulin A nephropathy

\begin{abstract}
Background/Aims: Stress-induced cell senescence, which contributes to cell cycle arrest and is independent of age, plays an important role in chronic kidney disease (CKD) progression. DcR2, as a senescent marker, exclusively expressed in senescent tubular epithelia. The objective of this study was to examine whether urinary DcR2 (uDcR2) could be a potential biomarker for tubulointerstitial fibrosis (TIF) in patients with immunoglobulin A nephropathy (IgAN). Methods: This study included 210 IgAN patients and 80 healthy volunteers, with uDCR2 levels measured using enzyme-linked immunosorbent assay. We examined the relationship among $\mathrm{uDCR} 2 / \mathrm{Cr}$ levels, renal function, and pathological parameters, using regression analysis to identify risk factors for TIF and the area under the curve (AUC) approach to predict TIF. Renal DcR2 expression was quantified by immunohistochemistry. Co-expression of DcR2 with fibrotic markers ( $\alpha$-smooth muscle actin $[\alpha-S M A]$, collagen III) was analyzed by confocal microscopy. Results: Levels of uDcR2/Cr were significantly higher in IgAN patients and in those with more severe TIF, compared with healthy controls. Serum DcR2 levels were similar across groups. The proportion of IgAN patients with stages 1-2 CKD and T0 was highest among those with uDcR2/Cr $<130 \mathrm{ng} / \mathrm{g}$. In contrast, the majority of those with uDcR2/Cr $>201 \mathrm{ng} / \mathrm{g}$ had stages 4-5 CKD and T2. Levels of $u D c R 2 / C r$ were positively associated with urinary albumin to creatinine ratio (ACR), urinary $N$-acetyl- $\beta$-D-glucosaminidase ( $U N A G) / C r$, and TIF scores
\end{abstract}

J.C. and W.H. contributed equally to this work. 


\section{Kidney \\ Blood Pressure \\ Research}

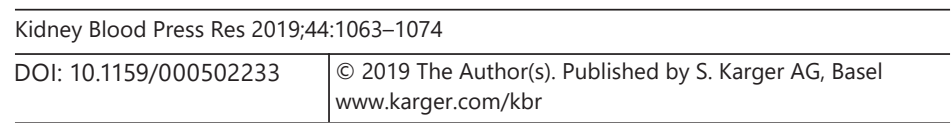

Chen et al.: DCR2, a Cellular Senescent Molecule, Is a Novel Marker for Assessing TIF in Patients with IgAN

and negatively associated with estimated glomerular filtration rate (eGFR). UDcR2/Cr, uNAG, $A C R$, and eGFR were independent predictors for TIF, with AUC of 0.907 for $\mathrm{UDCR} 2 / \mathrm{Cr}$. This AUC value was higher than that observed for eGFR, uNAG/Cr, or ACR. The sensitivity and specificity of $\mathrm{UDCR} 2 / \mathrm{Cr}$ in predicting TIF were 87.0 and $80.5 \%$, respectively. Moreover, $\mathrm{UDCR} 2 / \mathrm{Cr}$ levels were positively associated with the percentage of renal DcR2 expression. Renal DcR2 colocalized with $\alpha$-SMA and collagen III in the kidneys of IgAN patients. Conclusions: Levels of $\mathrm{UDCR} 2 / \mathrm{Cr}$ were closely associated with the severity of TIF and renal function parameters. $\mathrm{uDcR} 2 / \mathrm{Cr}$ represents a potential biomarker for predicting TIF in IgAN patients.

(C) 2019 The Author(s)

Published by S. Karger AG, Basel

\section{Introduction}

Immunoglobulin A nephropathy (IgAN) is the most common primary glomerulonephritis in young adults $[1,2]$. About $20-40 \%$ of patients with IgAN experience deterioration of renal function, progressing to end-stage renal disease within 20 years [3]. Evidence suggests that tubulointerstitial injury is an independent risk factor for the progression of IgAN and prominently affects patient prognosis [4]. During clinical practice, tubulointerstitial injury is assessed predominantly by pathological examination of renal biopsy specimens. However, renal biopsy is an invasive procedure, and repetitive biopsy can be difficult. Traditional renal function markers, including proteinuric amount and estimated glomerular filtration rate (eGFR), typically estimate the extent of injury to the glomeruli but not to the tubulointerstitium. Therefore, it is imperative to identify a noninvasive biomarker capable of assessing the severity of tubulointerstitial injury [5-10].

Stress-induced cell senescence denotes the process of an age-independent cell cycle arrest, induced by various pathological stimuli. Cellular senescence can result in irreversible tissue damage and organ dysfunction $[11,12]$. Senescent cells can secrete various senescence-associated secretory phenotypes, including proinflammatory factors and chemokines, which accelerate the progression of tissue damage and play a significant role in diabetes, cardiovascular disease, and chronic kidney disease (CKD) [11, 12]. CKD has been considered as a clinical model of premature senescence [11]. Previously, we found that tubular epithelia expressed senescent markers such as p16, p21, and senescence-associated- $\beta$-galactosidase, which were closely associated with the severity of tubulointerstitial injury and poorer renal function among young patients with IgAN [13].These findings indicate that cellular senescence plays an important role in the pathogenesis of tubulointerstitial injury in patients with IgAN. Uncovering a clinically useful biomarker to evaluate the severity of tubulointerstitial injury based on the extent of cellular senescence may be a promising approach.

DcR2, a decoy receptor for tumor necrosis factor-related apoptosis-inducing ligand, is a marker of senescence $[5,6,14]$. Previous studies found that DcR2 was highly expressed in senescent tumor cells and was associated with the degree of tumor differentiation [5, 6]. DcR2 is also expressed in activated senescent hepatic stellate cell, which protects senescent cell from cytotoxicity, eventually resulting in liver fibrosis [6]. Our laboratory previously found that DcR2 was exclusively expressed in renal tubular epithelia and co-expressed with senescent markers in patients with diabetic nephropathy, and the extracellular portion of DcR2 was detectable in urine $[15,16]$. In this study, we aimed to measure the ratio of urinary DcR2 to urinary creatinine (uDcR2/Cr) and renal DcR2, to evaluate the relationship between DcR2 expression and the severity of tubulointerstitial injury in IgAN patients. We hypothesized that uDcR2/Cr could be a biomarker for predicting the severity of tubulointerstitial injury in IgAN patients. 


\section{Kidney \\ Blood Pressure \\ Research}

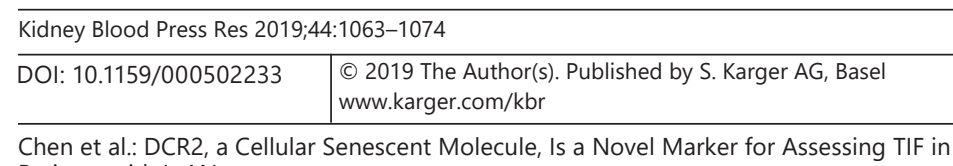
Patients with IgAN

\section{Methods}

\section{Ethical Statement}

We have obtained approval from the Ethical Committee of the Army Military Medical University and collected written informed consent from all participants.

Clinical Characteristics of Study Participants and the Origin of Biopsy Samples

We included 210 patients, aged from 18 to 65 years, with biopsy-proven IgAN. Patients with secondary IgAN (e.g., Henoch-Schönlein purpura nephritis, hepatitis B virus-associated glomerulonephritis) were excluded, as were patients with other types of renal diseases (e.g., diabetic nephropathy) and those with severe medical illnesses (e.g., neoplasm, severe infection, heart failure) [17]. The control group consisted of age-matched healthy volunteers $(n=80)$. Normal kidney tissue from areas adjacent to renal hamartoma was resected from otherwise healthy controls $(n=10)$ for analysis.

\section{Pathological Classification and Histological Scoring of IgAN}

Two pathologists assessed the sections according to the Oxford classification of IgAN [18], based on the following criteria: $\mathrm{T}$ (tubular atrophy/interstitial fibrosis $\leq 25 \%$, $\mathrm{T} 0$; 25-50\%, T1; >50\%, T2); E (endocapillary hypercellularity); S (segmental sclerosis); C (crescents); hyaline changes in arteries; and M (mesangial hypertrophy).

Measurement of DcR2 Levels in Blood and Urine with Enzyme-Linked Immunosorbent

Assay

We collected blood and urine from patients with IgAN, 1 day before renal biopsy. In the control group, blood and urine were collected from samples obtained during health checkups. Supernatant harvested from collected samples after centrifugation at 1,000 g for $15 \mathrm{~min}$ were stored at $-80^{\circ} \mathrm{C}$. Specimens were diluted 5 times. DcR2 levels were measured with a sandwich enzyme-linked immunosorbent assay kit (Abcam), according to the manufacturer's instructions. uDcR2 levels were calibrated to urinary $\mathrm{Cr}$ to account for sample concentration [19].

Measurement of Urinary $N$-acetyl- $\beta$-D-glucosaminidase and Other Parameters

Urinary $\mathrm{N}$-acetyl- $\beta$-D-glucosaminidase (UNAG) level was measured with a spectrophotometer calibrated to urinary $\mathrm{Cr}$ level, as described in our previous report (Chen et al. [16]). Biochemical parameters were measured with an automatic chemistry analyzer (Beckman). eGFR was calculated using the CKD - Epidemiology Collaboration equation [20].

\section{Immunohistochemical Staining for Renal DcR2 Expression}

Renal biopsy specimens, at a thickness of $2.5 \mu \mathrm{m}$, were treated with a mouse DcR2 monoclonal antibody (Abcam) as the primary antibody (1:400). Two pathologists blinded to the study results independently assessed the specimens [21] and calculated the percentages of tubular epithelia with positive DcR2 staining in 10 randomly selected highpower fields.

\section{Immunofluorescence Double Staining}

DcR2 were co-stained with $\alpha$-smooth muscle actin ( $\alpha$-SMA) and collagen III (Abcam). The primary antibody for DcR2 was bound by a Cy3-labeled secondary antibody (Abcam), while $\alpha$-SMA and collagen III were bound by a FITC-labeled secondary antibody (Abcam). Images were obtained by a laser scanning confocal microscope (Leica). Percentages of tubular epithelia with positive staining for DcR2 and $\alpha$-SMA were calculated. 


\section{Kidney \\ Blood Pressure \\ Research}

\begin{tabular}{|c|c|}
\hline \multicolumn{2}{|c|}{ Kidney Blood Press Res 2019;44:1063-1074 } \\
\hline DOI: 10.1159/000502233 & $\begin{array}{l}\text { (c) } 2019 \text { The Author(s). Published by S. Karger AG, Basel } \\
\text { www.karger.com/kbr }\end{array}$ \\
\hline
\end{tabular}

Chen et al.: DCR2, a Cellular Senescent Molecule, Is a Novel Marker for Assessing TIF in Patients with IgAN

Table 1. Demographics and clinical characteristics of slow and fast TAC metabolizers at all timepoints $(3,6,12$ and 24 months after kidney transplantation)

\begin{tabular}{|c|c|c|c|}
\hline & $\begin{array}{l}\text { Slow metabolizers } \\
n=50\end{array}$ & $\begin{array}{l}\text { Fast metabolizers } \\
n=51\end{array}$ & $p$ \\
\hline Age at baseline, years & $49.0 \pm 12.2$ & $48.3 \pm 13.3$ & 0.765 \\
\hline Sex, M/F & $31(60.8 \%) / 20(39.2 \%)$ & $31(62.0 \%) / 19(38.0 \%)$ & 0.900 \\
\hline Donor age, years & $47.6 \pm 13.0$ & $44.6 \pm 12.4$ & 0.253 \\
\hline Donor sex, M/F & $32(62.7 \%) / 19(37.3 \%)$ & $28(56.0 \%) / 22(44.0 \%)$ & 0.490 \\
\hline Type 2 Diabetes Mellitus & $6(11.8 \%)$ & $2(4.0 \%)$ & 0.154 \\
\hline New onset diabetes mellitus & $12(23.5 \%)$ & $10(20.0 \%)$ & 0.627 \\
\hline \multicolumn{4}{|l|}{3 months post $\mathrm{KTx}$} \\
\hline TAC daily dose, mg & $6.8 \pm 4.0$ & $10.6 \pm 4.7$ & $<0.001^{*}$ \\
\hline TAC $\mathrm{C}_{\mathrm{p} \text { trough }}, \mathrm{ng} / \mathrm{mL}$ & $10.83 \pm 3.68$ & $9.71 \pm 3.12$ & 0.105 \\
\hline $\mathrm{C} / \mathrm{D}$ ratio, $\mathrm{ng} / \mathrm{mL}^{*} 1 / \mathrm{mg}$ & $1.28 \pm 0.96$ & $1.06 \pm 0.54$ & 0.172 \\
\hline Creatinine concentration, $\mathrm{mg} / \mathrm{dL}$ & $1.55 \pm 0.48$ & $2.02 \pm 1.12$ & $0.006^{*}$ \\
\hline $\mathrm{eGFR}, \mathrm{mL} / \mathrm{min} / 1.73 \mathrm{~m}^{2}$ & $47.91(36.73-63.82)$ & $40.05(30.16-51.40)$ & $0.015^{*}$ \\
\hline \multicolumn{4}{|l|}{6 months post $\mathrm{KTx}$} \\
\hline TAC daily dose, mg & $3.6 \pm 1.4$ & $7.7 \pm 4.3$ & $<0.001^{*}$ \\
\hline TAC $C_{p \text { trough }}, \mathrm{ng} / \mathrm{mL}$ & $8.30 \pm 2.38$ & $6.70 \pm 2.40$ & $0.001^{*}$ \\
\hline $\mathrm{C} / \mathrm{D}$ ratio, $\mathrm{ng} / \mathrm{mL}^{*} 1 / \mathrm{mg}$ & $2.52 \pm 1.03$ & $1.00 \pm 0.33$ & $<0.001^{*}$ \\
\hline Creatinine concentration, $\mathrm{mg} / \mathrm{dL}$ & $1.39 \pm 0.42$ & $1.72 \pm 0.61$ & $0.002^{*}$ \\
\hline $\mathrm{eGFR}, \mathrm{mL} / \mathrm{min} / 1.73 \mathrm{~m}^{2}$ & $58.46(42.33-72.71)$ & $49.45(32.97-57.77)$ & $0.002^{*}$ \\
\hline \multicolumn{4}{|l|}{12 months post KTx } \\
\hline TAC daily dose, mg & $3.1 \pm 1.2$ & $5.9 \pm 2.8$ & $<0.001^{*}$ \\
\hline TAC $C_{p \text { trough }}, n g / m L$ & $6.25 \pm 1.98$ & $6.61 \pm 2.61$ & 0.434 \\
\hline $\mathrm{C} / \mathrm{D}$ ratio, $\mathrm{ng} / \mathrm{mL}^{*} 1 / \mathrm{mg}$ & $2.32 \pm 1.18$ & $1.39 \pm 1.18$ & $<0.001^{*}$ \\
\hline Creatinine concentration, $\mathrm{mg} / \mathrm{dL}$ & $1.31 \pm 0.38$ & $1.62 \pm 0.58$ & $0.002^{*}$ \\
\hline $\mathrm{eGFR}, \mathrm{mL} / \mathrm{min} / 1.73 \mathrm{~m}^{2}$ & $61.21(48.42-73.65)$ & $51.82(36.95-63.36)$ & $0.002^{*}$ \\
\hline \multicolumn{4}{|l|}{24 months post KTx } \\
\hline TAC daily dose, mg & $2.7 \pm 1.0$ & $5.1 \pm 2.5$ & $<0.001^{*}$ \\
\hline TAC $\mathrm{C}_{\mathrm{p} \text { trough }}, \mathrm{ng} / \mathrm{mL}$ & $5.76 \pm 1.69$ & $6.08 \pm 1.79$ & 0.379 \\
\hline $\mathrm{C} / \mathrm{D}$ ratio, $\mathrm{ng} / \mathrm{mL}^{*} 1 / \mathrm{mg}$ & $2.56 \pm 1.44$ & $1.46 \pm 0.80$ & $<0.001^{*}$ \\
\hline Creatinine concentration, $\mathrm{mg} / \mathrm{dL}$ & $1.27 \pm 0.38$ & $1.54 \pm 0.49$ & $0.005^{*}$ \\
\hline $\mathrm{eGFR}, \mathrm{mL} / \mathrm{min} / 1.73 \mathrm{~m}^{2}$ & $64.83(53.04-77.32)$ & $48.77(39.71-61.44)$ & $0.006^{*}$ \\
\hline
\end{tabular}

Nominal variables are presented as absolute and relative numbers and continuous data are presented as means with standard deviations or as medians with the values of upper and lower quartile. Data for the 3rd, 6th and 12th month after kidney transplantation are presented for 101 patients (slow metabolizers: $n=51$, fast metabolizers: $n=50$ ), data for the 24th month are presented for 90 patients (slow metabolizers: $n=45$, fast metabolizers: $n=45$ ). eGFR, estimated glomerular filtration rate; $\mathrm{TAC}$, tacrolimus; $\mathrm{TAC} \mathrm{C}_{\mathrm{p} \text { trough }}$ TAC trough concentration.

\section{Statistical Analyses}

Statistical analyses were conducted using SPSS version 20.0. Normally distributed data were described as and analyzed using one-way analysis of variance. Nonnormally distributed data were described as medians with interquartile ranges in parentheses and analyzed using the Kruskal-Wallis test. Count data were described as frequency (with percentage) and analyzed using the chi-square test. Analyses of correlations between uDcR2 levels and renal function markers were performed using Pearson's correlation or Spearman's rank-order correlation. We used logistic regression to identify risk factors for tubulointerstitial was used to evaluate the sensitivity and specificity of uDcR2 as a marker for predicting TIF. $p<0.05$ was considered significant. 
Table 2. Levels of uDcR2/Cr, uNAG/Cr, and serum DcR2 in IgAN patients

\begin{tabular}{|c|c|c|c|c|c|}
\hline \multirow[t]{2}{*}{ Clinical data } & \multirow{2}{*}{$\begin{array}{l}\text { Control } \\
(n=80)\end{array}$} & \multirow{2}{*}{$\begin{array}{l}\text { IgAN patients } \\
(n=210)\end{array}$} & \multicolumn{3}{|c|}{ Tubular scores } \\
\hline & & & T0 $(n=120)$ & $\mathrm{T} 1(n=71)$ & $\mathrm{T} 2(n=19)$ \\
\hline uDcR2/Cr, ng/g Cr & $56.9 \pm 23.2$ & $169.7 \pm 76.6^{*}$ & $102.2 \pm 53.5^{*}$ & $176.6 \pm 86.4^{\#}$ & $256.7 .6 \pm 108.6^{\#, \wedge}$ \\
\hline uNAG/Cr, U/g Cr & $5.6 \pm 4.3$ & $20.8 \pm 8.5^{*}$ & $9.8 \pm 6.3^{*}$ & $19.8 \pm 9.6^{\#}$ & $25.8 \pm 13.2^{\#, \wedge}$ \\
\hline Serum DcR2, pg/mL & $204.9 \pm 67.1$ & $220.1 \pm 60.4$ & $219.3 \pm 56.3$ & $226.1 \pm 59.1$ & $237.1 \pm 71.0$ \\
\hline
\end{tabular}

${ }^{*} p<0.05$ vs. control; ${ }^{*} p<0.05$ vs. T0; ${ }^{\wedge} p<0.05$ vs. T1.

uDcR2, urinary DcR2; uNAG, urinary N-acetyl- $\beta$-D-glucosaminidase; IgAN, immunoglobulin A nephropathy.

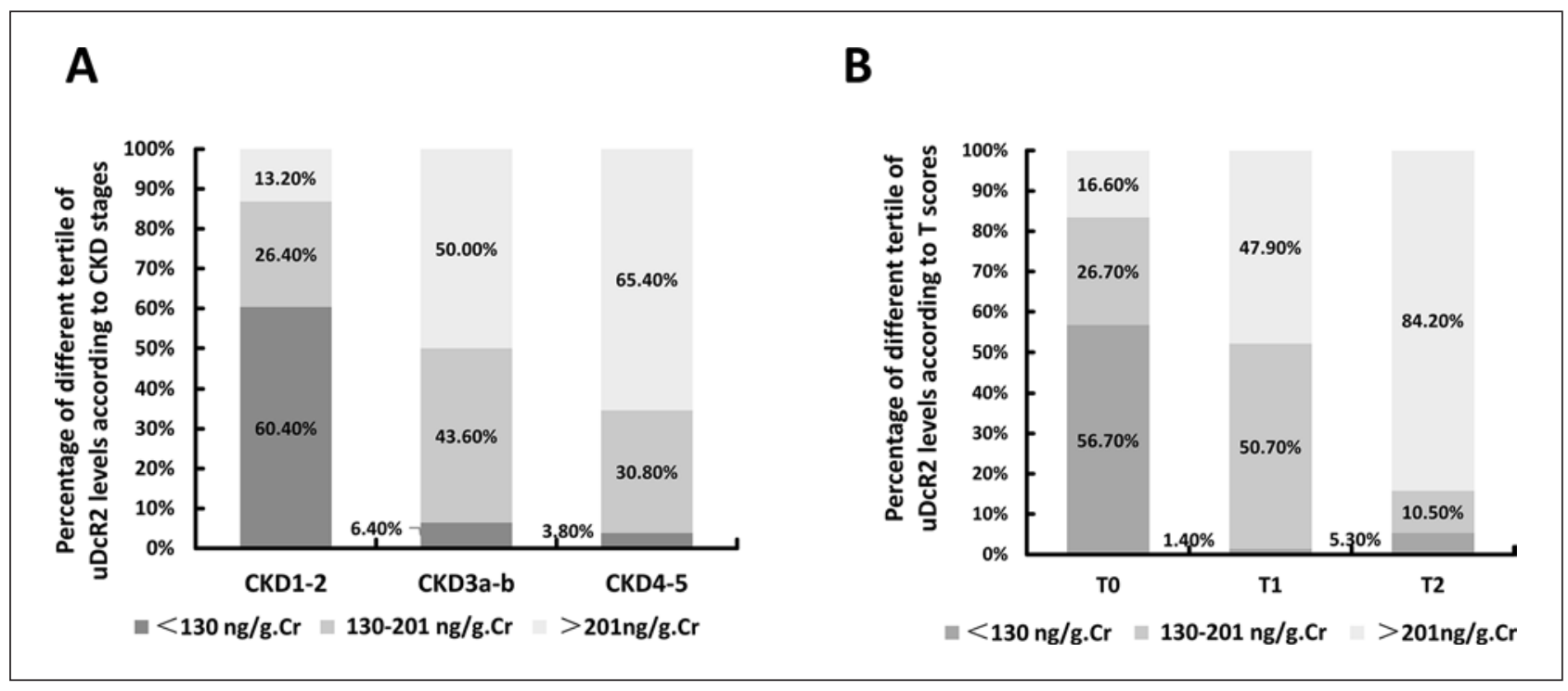

Fig. 1. uDcR2 levels in IgAN patients with different CKD stages (A) and severities of tubulointerstitial injury (B). CKD, chronic kidney disease; uDcR2, urinary DcR2; Cr, creatinine.

\section{Results}

Clinical Characteristics of Healthy Controls and Patients with IgAN

Clinical features of 210 subjects with IgAN and those of 80 healthy controls are shown in Table 1. Compared with the healthy controls, IgAN patients had higher systolic blood pressure, serum uric acid (UA), Cr, and urinary albumin to $\mathrm{Cr}$ ratio (ACR) levels but lower serum albumin, hemoglobin, and eGFR levels (all $p<0.05$ ). There was no difference in age, gender, body mass index, or diastolic blood pressure between the 2 groups. IgAN patients were further classified into 3 subgroups: T0 $(n=120), \mathrm{T} 1(n=71)$, and T2 $(n=19)$, based on the T scores of the Oxford classification of IgAN. We found that with higher T scores, IgAN patients also had higher systolic blood pressure, serum UA, Cr, and urinary ACR levels, but lower hemoglobin and eGFR.

\section{$u D c R 2 / C r$ and $u N A G / C r$ and Serum DcR2 Levels in Patients with IgAN}

uDcR2/Cr and uNAG/Cr levels were significantly higher in patients with IgAN, compared with those in healthy controls. Among those with higher T scores, uDcR $2 / \mathrm{Cr}$ and $\mathrm{uNAG} / \mathrm{Cr}$ levels were also higher among patients with IgAN; however, there was no significant difference in serum DcR2 levels between healthy controls and IgAN patients (Table 2). 
Table 3. Correlations between uDcR2/Cr levels and clinical/histopathological parameters of patients with IgAN

\begin{tabular}{lcc}
\hline Clinical/histopathological parameters & \multicolumn{2}{l}{ uDcR2/Cr } \\
\cline { 2 - 3 } & $r$ value & $p$ value \\
\hline Clinical parameters & & \\
eGFR, mL/min/1.73 m ${ }^{2}$ & -0.626 & 0.000 \\
UA, $\mu \mathrm{mol} / \mathrm{L}$ & 0.513 & 0.000 \\
ACR, mg/g Cr & 0.646 & 0.000 \\
uNAG, U/g Cr & 0.617 & 0.000 \\
Histopathological parameters & & \\
Tubular atrophy/interstitial fibrosis & 0.692 & 0.000 \\
$\quad$ Segmental glomerulosclerosis and adhesions & 0.437 & 0.008 \\
Mesangial proliferation (M) & 0.211 & 0.239 \\
Endocapillary proliferation (E) & 0.287 & 0.213 \\
Cresent (C) & 0.337 & 0.096 \\
Glomerulosclerosis & 0.397 & 0.015 \\
Interstitial infiltration & 0.605 & 0.001 \\
Arteriolar hyalinosis & 0.180 & 0.310 \\
\hline
\end{tabular}

uDcR2/Cr, urinary DcR2/creatinine; IgAN, immunoglobulin A nephropathy; eGFR, estimated glomerular filtration rate; ACR, albumin to creatinine ratio; uNAG, urinary $\mathrm{N}$-acetyl- $\beta$-D-glucosaminidase; UA, uric acid.

Distribution of IgAN Patients with Different CKD Stages and T Scores According to the

Tertile of uDcR2/Cr Levels

Patients with IgAN were classified into 3 subgroups based on their uDcR2/Cr level: $<130$, $130-210$, and $>210 \mathrm{ng} / \mathrm{g}$. With higher CKD stage, IgAN patients had higher uDcR2/Cr levels. uDcR2/Cr levels similarly increased in association with T scores (Fig. 1).

Correlations between $\mathrm{UDCR} 2 / \mathrm{Cr}$ Levels and Clinical and Histopathological Parameters

We observed positive associations between uDcR2/Cr levels and serum UA, urinary ACR, and $\mathrm{UNAG} / \mathrm{Cr}$, and pathological findings including the severity of tubular atrophy/interstitial fibrosis, interstitial inflammation, glomerulosclerosis, and segmental glomerular hypercellularity/adhesion. A negative correlation was found between uDcR2/Cr levels and eGFR (Table 3).

\section{Logistic Regression Analysis to Identify Risk Factors for TIF}

We analyzed the clinical and pathological features of IgAN patients to identify determinants for TIF $>25 \%$ using logistic regression analyses (Table 4). Univariate analysis showed that higher serum UA, eGFR, urinary ACR, uDcR2/Cr, and uNAG/Cr were associated with TIF $>25 \%$, while higher baseline eGFR was associated with decreased risk for TIF. Multivariate logistic regression analysis showed that ACR (OR 2.11, $p=0.007$ ), eGFR (OR 0.97, $p=0.019$ ), uDcR2/Cr (OR 3.23, $p<0.001$ ), and uNAG (OR 1.55, $p=0.032$ ) levels were independent predictors for TIF $>25 \%$ among IgAN patients. Based on the abovementioned risk factors for TIF (eGFR as X1, ACR as X2, uDcR2/Cr as X3, and uNAG/Cr as X4), we constructed the regression model as follows:

Logit $(\mathrm{P})=-5.953-1.361^{*} \mathrm{eGFR}+1.404^{*} \mathrm{ACR}+1.674^{*} \mathrm{uDcR} 2 / \mathrm{Cr}+1.201^{*} \mathrm{uNAG}$

Using this regression formula, the possibility of having TIF $>25 \%$ among IgAN patients could be inferred using known eGFR, urinary ACR, uDcR2/Cr, and UNAG values. 
Table 4. Risk factors for TIF by univariate and multivariate logistic regression

\begin{tabular}{|c|c|c|c|c|}
\hline & \multicolumn{2}{|l|}{ Univariate } & \multicolumn{2}{|l|}{ Multivariate } \\
\hline & OR $(95 \% \mathrm{CI})$ & $p$ value & OR (95\% CI) & $p$ value \\
\hline Gender, male & $1.13(0.89-3.78)$ & 0.671 & & \\
\hline Age, years & $1.0(0.97-1.04)$ & 0.830 & & \\
\hline Hypertension & $1.64(0.92-2.68)$ & 0.047 & $1.07(0.69-1.80)$ & 0.207 \\
\hline $\mathrm{Hb}$ & $0.82(0.67-0.98)$ & 0.066 & & \\
\hline Alb & $0.78(0.24-0.67)$ & 0.091 & & \\
\hline UA & $1.75(1.38-2.17)$ & 0.033 & $1.02(0.59-1.50)$ & 0.795 \\
\hline eGFR (X1) & $0.92(0.90-0.96)$ & 0.001 & $0.97(0.93-0.99)$ & 0.019 \\
\hline ACR (X2) & $3.28(1.61-4.36)$ & 0.000 & $2.11(1.36-3.58)$ & 0.007 \\
\hline uDcR2/Cr (X3) & $4.70(2.87-8.67)$ & 0.000 & $3.23(2.18-5.62)$ & 0.000 \\
\hline uNAG/Cr (X4) & $1.992(1.07-3.71)$ & 0.030 & $1.55(1.16-2.67)$ & 0.032 \\
\hline
\end{tabular}

TIF, tubulointerstitial fibrosis; Alb, albumin; Hb, hemoglobin; UA, uric acid; eGFR, estimated glomerular filtration rate; ACR, albumin to creatinine ratio; uDcR2, urinary DcR2; uNAG, urinary N-acetyl- $\beta$-Dglucosaminidase; $\mathrm{Cr}$, creatinine.

Fig. 2. ROC curve analysis of uDcR2/Cr levels and uNAG/Cr for assessing TIF in IgAN patients. uNAG, urinary N-acetyl- $\beta$-D-glucosaminidase; uDcR $2 / \mathrm{Cr}$, urinary DcR2/creatinine.

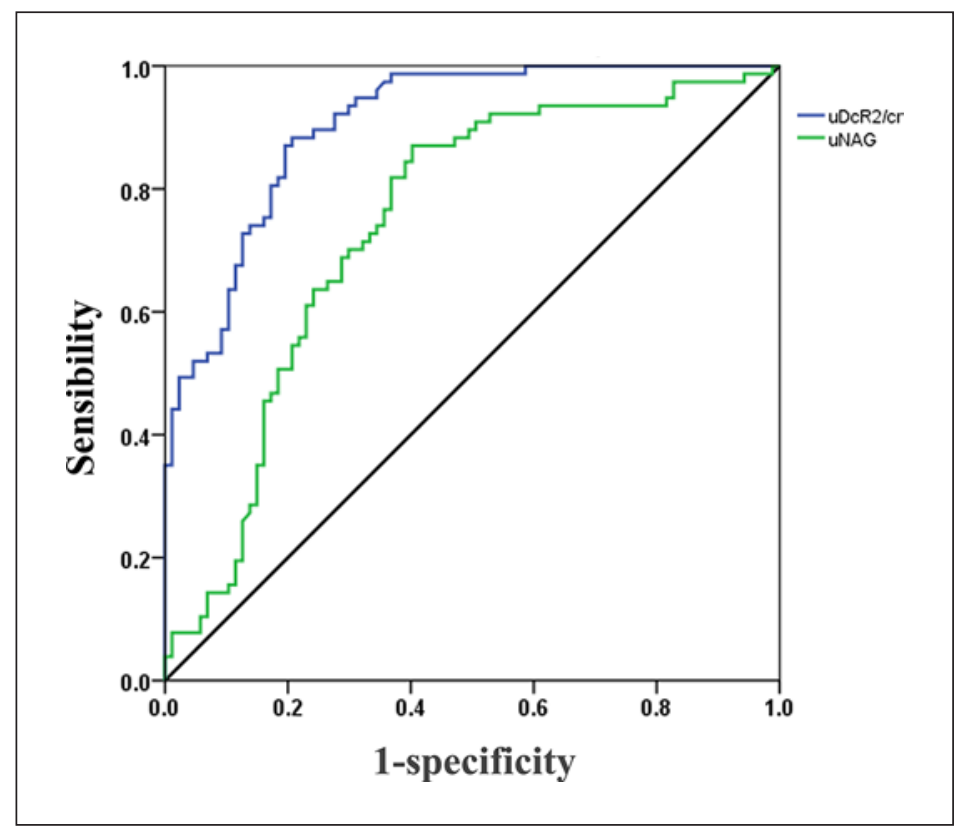

ROC Curve Analysis of uDcR2/Cr and uNAG/Cr for Predicting TIF in IgAN Patients

IgAN patients were further classified into 2 groups based on the presence or absence of TIF (presence vs. absence, 120 vs. 90), and we applied the ROC curves to estimate the sensitivity and specificity of using $\mathrm{uDcR} / \mathrm{Cr}$ to predict TIF. We found that area under the curve was 0.907 for $\mathrm{uDcR} / \mathrm{Cr}$, which is significantly higher than that for traditional TIF marker NAG (area under the curve =0.746; Fig. 2). The best cutoff for diagnosing TIF was $156.82 \mathrm{ng} / \mathrm{g}$, with sensitivity and specificity of 87 and $80.5 \%$, respectively. 


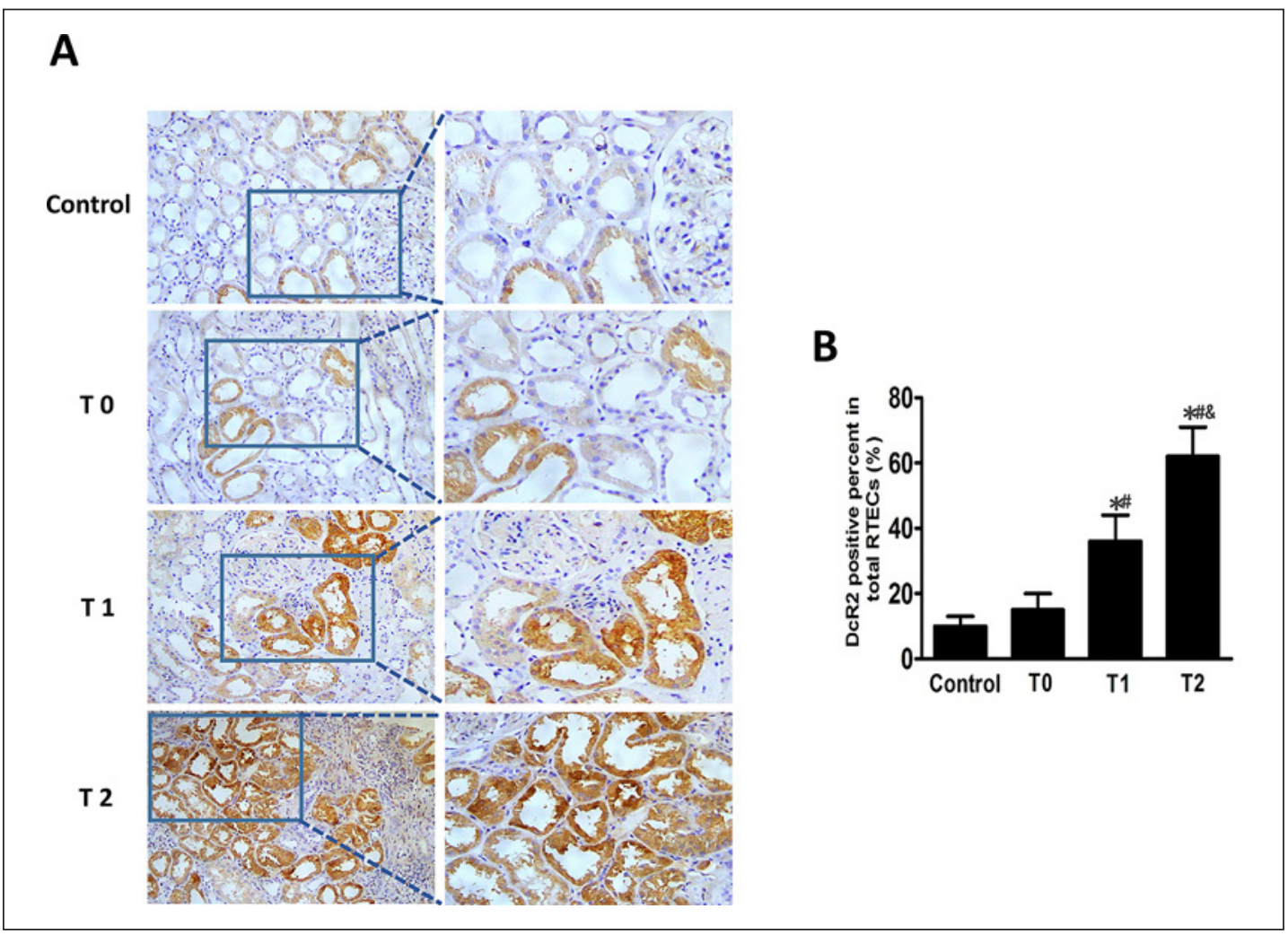

Fig. 3. Immunohistochemical staining of DcR2 expression in patients with IgAN and healthy controls (A; $\times 100$ ) and the percentage of DcR2 expression in renal tubular epithelial cells (RTECs) (B). * $p<0.05$, versus control; ${ }^{\#} p<0.05$, versus T0; ${ }^{\&} p<0.05$, versus T1.

Fig. 4. Correlation analyses between tubular DcR2 expression levels and $u D c R 2 / C r$ levels in IgAN patients. uDcR $2 / \mathrm{Cr}$, urinary DcR2/creatinine; RTECs, renal tubular epithelial cells.

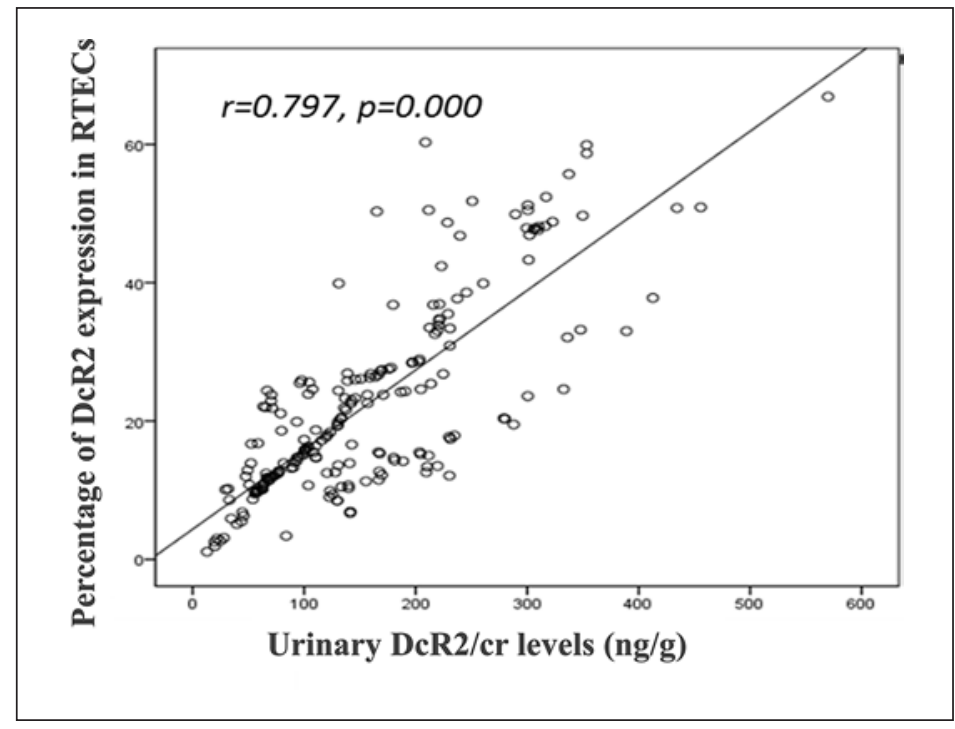

Tubular DcR2 Expression and Its Correlation with uDcR2/Cr in IgAN Patients

Immunohistochemistry staining showed that DcR2 was exclusively expressed in renal tubular epithelial cells (RTECs) but not glomeruli (Fig. 3A). Compared with the healthy controls, IgAN patients had significantly higher renal DcR2 expression (Fig. 3B). In addition, 
Kidney

Blood Pressure

Research

Fig. 5. Co-expression of DcR2 and fibrotic markers in renal tissue from IgAN patients. A Co-expression of DcR2 and $\alpha$-SMA. B, C The percentage of positive-staining cells. D Co-expression of DcR2 and collagen III in renal tissue. * $p<0.05$, versus control; \# $p<$ 0.05 , versus $\mathrm{T} 0$; $^{\&} p<0.05$, versus T1. $\alpha$-SMA, $\alpha$-smooth muscle actin;

\begin{tabular}{l|l}
\hline Kidney Blood Press Res 2019:44:1063-1074 \\
\hline DOI: 10.1159/000502233 & $\begin{array}{l}\text { ○ 2019 The Author(s). Published by S. Karger AG, Basel } \\
\text { www.karger.com/kbr }\end{array}$ \\
\hline
\end{tabular}

Chen et al.: DCR2, a Cellular Senescent Molecule, Is a Novel Marker for Assessing TIF in Patients with IgAN

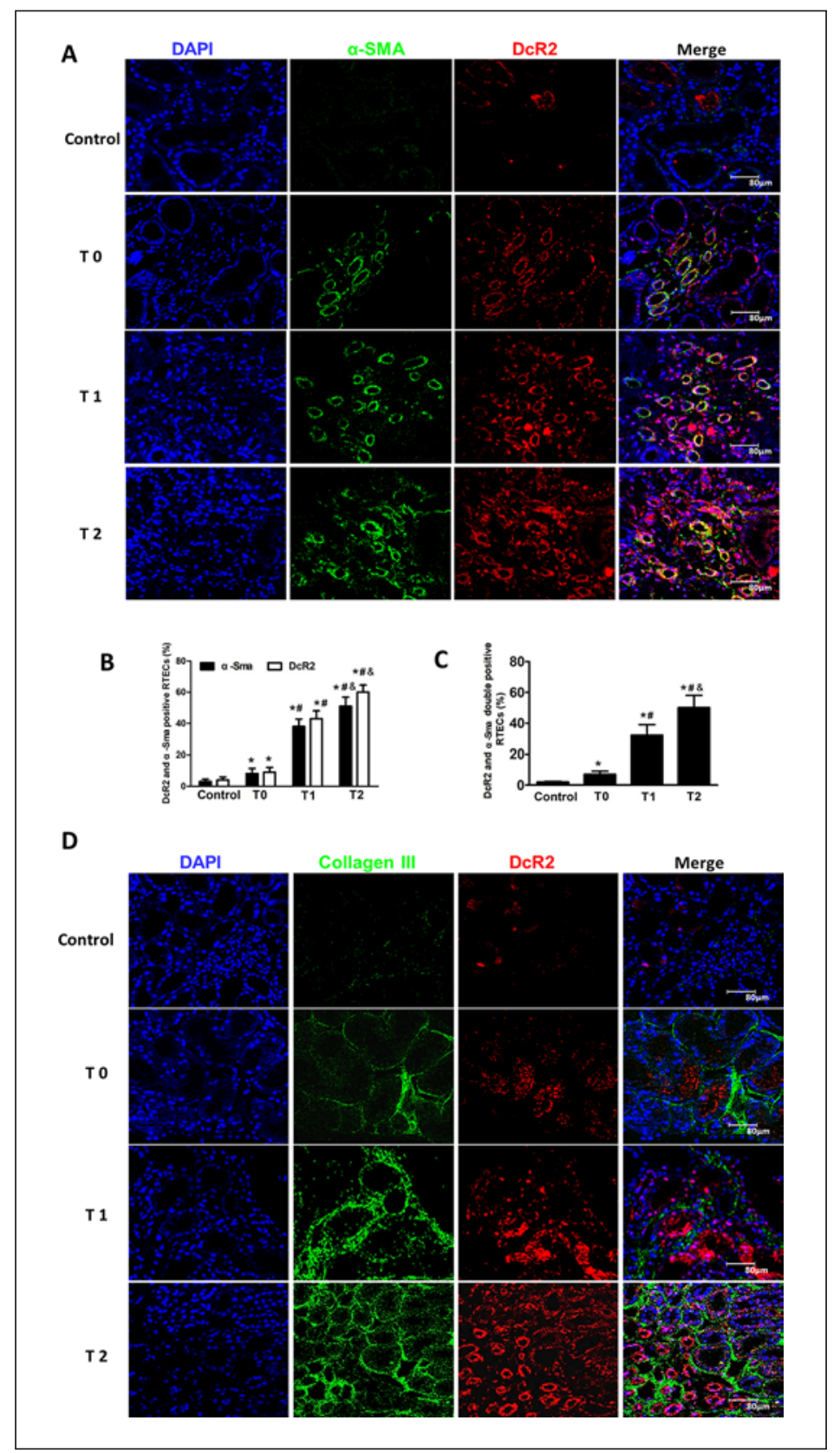

uDcR2/Cr level was positively correlated with the percentage of RTECs positively stained for DcR2 ( $r=0.797, p<0.001$; Fig. 4).

\section{Co-expression of DcR2 and Fibrotic Markers in Renal Tissue}

The expressions of $\alpha$-SMA and collagen III increased with higher TIF scores in IgAN patients. Immunofluorescence double staining showed that DcR2 was mainly expressed in areas that stained positive for $\alpha$-SMA and collagen III (Fig. 5A, D). We also found that higher TIF scores were associated with increasing percentages of doubly positive-staining cells (Fig. 5B, C). From these findings, we inferred that DcR2-positive senescent epithelia may contribute to TIF and worsen the outcomes of IgAN patients. 


\section{Kidney \\ Blood Pressure \\ Research}

\begin{tabular}{l|l}
\hline Kidney Blood Press Res 2019:44:1063-1074 \\
\hline DOI: 10.1159/000502233 & $\begin{array}{l}\text { ○ 2019 The Author(s). Published by S. Karger AG, Basel } \\
\text { www.karger.com/kbr }\end{array}$ \\
\hline
\end{tabular}

Chen et al.: DCR2, a Cellular Senescent Molecule, Is a Novel Marker for Assessing TIF in Patients with IgAN

\section{Discussion}

TIF is an important outcome predictor among patients with IgAN [22]. Urine biomarkers including NAG, kidney injury molecule-1, and neutrophil gelatinase-associated lipocalin have been utilized for the evaluation of tubulointerstitial injury [14, 23-25], but these biomarkers cannot be used to determine the extent of tubular proliferation and repair. Prior studies suggested that stress-induced senescence of tubular epithelia was pathophysiologically important in the course of IgAN. Senescent cells may cause damage to healthy cells in adjacent areas, leading to local inflammation and fibrosis through the process of senescence-associated secretory phenotypes (including proinflammatory factors and chemokines). In addition, senescent cells can also suppress cellular proliferation and regeneration, leading to irreversible injury and a slowed repair process. In line with these data, we tried to identify biomarkers reflective of tubular repair and to determine whether there was a correlation with the severity of tubulointerstitial injury among patients with IgAN. Traditional markers of cellular senescence, such as $\mathrm{p} 16$ and p21, are mainly expressed in tubular cell nuclei and plasma, although these markers are not secreted and not detectable in the urine [26, 27]. In contrast, the extracellular portion of the senescence marker DcR2 can be cleaved and then detected in biologic fluid. Based on this assumption, we measured uDcR2/Cr levels among groups of IgAN patients and healthy volunteers and found that uDcR2/Cr levels were significantly higher among IgAN patients compared with healthy controls. Similarly, uDcR2/Cr levels increased with higher tubulointerstitial injury scores. Our findings suggest that $\mathrm{uDcR} 2 / \mathrm{Cr}$ could be a potential biomarker for tubulointerstitial injury among IgAN patients.

DcR2 is a $42-\mathrm{kDa}$ transmembrane protein and can be filtered freely by glomeruli, a property that may impact the $\mathrm{uDcR} 2 / \mathrm{Cr}$ level. We found that there was no difference in serum DcR2 levels between IgAN patients and healthy controls, while uDcR2 levels were significantly higher in IgAN patients, compared with healthy controls. These findings indicate that urine $\mathrm{uDcR} 2 / \mathrm{Cr}$ level can be a surrogate for the severity of kidney injury, similar to results obtained previously in our study of patients with diabetic nephropathy. In conclusion, uDcR2/ Cr might be a novel biomarker for TIF in patients with CKD. To uncover the source of uDcR2, we performed immunohistochemistry staining of renal tissues. The results showed that tubular epithelia but not glomerular cells expressed DcR2. It is likely that uDcR2/Cr comes directly from DcR2-expressing senescent tubular cells, and DcR2 is a potential marker for evaluating TIF.

Using multivariate logistic regression, we found that $\mathrm{uDcR} 2 / \mathrm{Cr}$ was an independent predictor of TIF. Previously, Machiguchi and colleagues discovered that uNAG/Cr could be a useful marker for predicting the progression of IgAN; levels correlated closely with both clinical and histological findings [28]. In another study, the sensitivity and specificity of uNAG/Cr for the diagnosis of Lee's grade III IgAN were 82.4 and 60.5\%, respectively [29]. Based on our findings, DcR2 could be a superior biomarker for IgAN. We further showed that DcR2 co-colocalized with fibrotic markers. These findings suggest that DcR2-positive senescent cells can increase the production of extracellular matrix and collagen III, resulting in renal fibrosis.

uDcR2/Cr could be a noninvasive biomarker for evaluating the severity of TIF among patients with IgAN, with significantly higher sensitivity and specificity than offered by traditional markers such as NAG. However, the current study is still limited in several ways. It is a cross-sectional single-center study, so prospective studies with a larger sample size are needed to confirm the utility of $\mathrm{uDcR} 2 / \mathrm{Cr}$ for diagnosis and evaluation of prognosis in patients with IgAN. In conclusion, uDcR2/Cr is a potential biomarker for accurately evaluating the severity of tubulointerstitial injury in IgAN. 


\section{Kidney \\ Blood Pressure \\ Research}

\begin{tabular}{l|l}
\hline Kidney Blood Press Res 2019;44:1063-1074 \\
\hline DOI: 10.1159/000502233 & $\begin{array}{l}\text { ○ 2019 The Author(s). Published by S. Karger AG, Basel } \\
\text { www.karger.com/kbr }\end{array}$ \\
\hline
\end{tabular}

Chen et al.: DCR2, a Cellular Senescent Molecule, Is a Novel Marker for Assessing TIF in Patients with IgAN

\section{Acknowledgments}

This study was financially supported by the National Natural Science Foundation of China (81800646, 81670661), the 12th 5-year Military Science Foundation (AWS14C003), and the National Science and Technology Support Plan (2015BAI12B06, 2016YFC1305501).

\section{Disclosure Statement}

All authors declared there were no conflicts of interest involved.

\section{References}

1 Suzuki H, Yasutake J, Makita Y, Tanbo Y, Yamasaki K, Sofue T, et al. IgA nephropathy and IgA vasculitis with nephritis have a shared feature involving galactose-deficient IgA1-oriented pathogenesis. Kidney Int. 2018 Mar; 93(3):700-5.

2 Wyatt RJ, Julian BA. IgA nephropathy. N Engl J Med. 2013 Jun;368(25):2402-14.

3 Zhang YM, Zhou XJ, Zhang H. What genetics tells us about the pathogenesis of iga nephropathy: the role of immune factors and infection. Kidney Int Rep. 2017 Feb;2(3):318-31.

4 Fabiano RC, Araújo SA, Bambirra EA, Oliveira EA, Simões E Silva AC, Pinheiro SV. The Oxford Classification predictors of chronic kidney disease in pediatric patients with IgA nephropathy. J Pediatr (Rio J). 2017 Jul Aug;93(4):389-97.

5 Flyvbjerg A. Diabetic angiopathy, the complement system and the tumor necrosis factor superfamily. Nat Rev Endocrinol. 2010 Feb;6(2):94-101.

6 Xue W, Zender L, Miething C, Dickins RA, Hernando E, Krizhanovsky V, et al. Senescence and tumour clearance is triggered by p53 restoration in murine liver carcinomas. Nature. 2007 Feb;445(7128):656-60.

7 Coppo R. Biomarkers and targeted new therapies for IgA nephropathy. Pediatr Nephrol. 2017 May;32(5): 725-31.

8 Hwang VJ, Ulu A, van Hoorebeke J, Weiss RH. Biomarkers in IgA nephropathy. Biomarkers Med. 2014;8(10): 1263-77.

9 Katafuchi R, Ninomiya T, Nagata M, Mitsuiki K, Hirakata H. Validation study of oxford classification of IgA nephropathy: the significance of extracapillary proliferation. Clin J Am Soc Nephrol. 2011 Dec;6(12):2806-13.

10 Maixnerova D, Reily C, Bian Q, Neprasova M, Novak J, Tesar V. Markers for the progression of IgA nephropathy. J Nephrol. 2016 Aug;29(4):535-41.

11 Salama R, Sadaie M, Hoare M, Narita M. Cellular senescence and its effector programs. Genes Dev. 2014 Jan; 28(2):99-114.

12 Sormani MP. Modeling the distribution of new MRI cortical lesions in multiple sclerosis longitudinal studies by Sormani MP, Calabrese M, Signori A, Giorgio A, Gallo P, De Stefano N [PLoS One 2011;6(10):e26712. Epub 2011 October 20]. Mult Scler Relat Disord. 2012 Jul;1(3):108.

13 Liu J, Yang JR, He YN, Cai GY, Zhang JG, Lin LR, et al. Accelerated senescence of renal tubular epithelial cells is associated with disease progression of patients with immunoglobulin A (IgA) nephropathy. Transl Res. 2012 Jun;159(6):454-63.

14 van Deursen JM. The role of senescent cells in ageing. Nature. 2014 May;509(7501):439-46.

15 Sanchez-Lazaro IJ, Almenar-Bonet L, Romero-Pelechano A, Portoles-Sanz M, Martinez-Dolz L, Rosello-Lleti E, et al. Serum markers of apoptosis in the early period of heart transplantation. Biomarkers. 2012 May;17(3): 254-60.

16 Chen J, Zhang WW, Chen KH, Lin LR, Dai HZ, Li KL, et al. Urinary DcR2 is a novel biomarker for tubulointerstitial injury in patients with diabetic nephropathy. Am J Physiol Renal Physiol. 2017 Aug;313(2):F273-81.

17 Yuzawa Y, Yamamoto R, Takahashi K, Katafuchi R, Tomita M, Fujigaki Y, et al. Evidence-based clinical practice guidelines for IgA nephropathy 2014. Clin Exp Nephrol. 2016 Aug;20(4):511-35.

18 Trimarchi H, Barratt J, Cattran DC, Cook HT, Coppo R, Haas M, et al.; IgAN Classification Working Group of the International IgA Nephropathy Network and the Renal Pathology Society. Conference Participants. Oxford classification of iga nephropathy 2016: an update from the iga nephropa-thy classification working group. Kidney Int. 2017 May;91(5):1014-21.

19 Hwang JH, Lee JP, Kim CT, Yang SH, Kim JH, An JN, et al. Urinary periostin excretion predicts renal outcome in iga nephropathy. Am J Nephrol. 2016;44(6):481-92.

20 Methven S, Gasparini A, Carrero JJ, Caskey FJ, Evans M. Routinely measured iohexol glomerular filtration rate versus creatinine-based estimated glomerular filtration rate as predictors of mortality in patients with advanced chronic kidney disease: a Swedish Chronic Kidney Disease Registry cohort study. Nephrol Dial Transplant. 2017 Apr;32 suppl_2:ii170-9. 
21 Du R, Zhao L, Xia L, Liu L, Sun W, Zhao A, et al. Association of URG11 and Twist with clinical pathological characteristics and prognosis in patients with IgA nephropathy. Nephrol Dial Transplant. 2013 Sep;28(9):226876.

22 Ferenbach DA, Bonventre JV. Acute kidney injury and chronic kidney disease: from the laboratory to the clinic. Nephrol Ther. 2016 Apr;12 Suppl 1:S41-8.

23 Lobato GR, Lobato MR, Thomé FS, Veronese FV. Performance of urinary kidney injury molecule-1, neutrophil gelatinase-associated lipocalin, and N-acetyl- $\beta$-D-glucosaminidase to predict chronic kidney disease progression and adverse outcomes. Braz J Med Biol Res. 2017 Mar;50(5):e6106.

24 Nickolas TL, Forster CS, Sise ME, Barasch N, Solá-Del Valle D, Viltard M, et al. NGAL (Lcn2) monomer is associated with tubulointerstitial damage in chronic kidney disease. Kidney Int. 2012 Sep;82(6):718-22.

25 Park HC, Hwang JH, Kang AY, Ro H, Kim MG, An JN, et al. Urinary N-acetyl- $\beta$-D glucosaminidase as a surrogate marker for renal function in autosomal dominant polycystic kidney disease: 1 year prospective cohort study. BMC Nephrol. 2012 Aug;13(1):93.

26 López-Otín C, Blasco MA, Partridge L, Serrano M, Kroemer G. The hallmarks of aging. Cell. 2013 Jun;153(6): 1194-217.

27 Muñoz-Espín D, Serrano M. Cellular senescence: from physiology to pathology. Nat Rev Mol Cell Biol. 2014 Jul; 15(7):482-96.

28 Machiguchi T, Yoshida H, Yonemoto S, Minakata T, Nomura K, Muso E, et al. Does circulating erythropoietin reflect progression of IgA nephropathy? Comparison with urinary N-acetyl-beta-D-glucosaminidase. Nephrol Dial Transplant. 1999 Mar;14(3):635-40.

29 Ding H, He Y, Li K, Yang J, Li X, Lu R, et al. Urinary neutrophil gelatinase-associated lipocalin (NGAL) is an early biomarker for renal tubulointerstitial injury in IgA nephropathy. Clin Immunol. 2007 May;123(2):227-34. 